\title{
Symmetry breaking in cyclic competition by niche construction
}

\author{
Xiaozhuo Han ${ }^{\mathrm{a}, \mathrm{b}, *}$, Baoying Chen ${ }^{\mathrm{a}}$, Cang Hui ${ }^{\mathrm{c}, \mathrm{d}}$ \\ a School of Applied Mathematics, Guangdong University of Technology, No. 161, Yinglong Road, Tianhe District, Guangzhou 510520, \\ PR China \\ ${ }^{\mathrm{b}}$ York Centre for Complex Systems Analysis, Ron Cooke Hub, University of York, York YO10 5GE, UK \\ c Centre for Invasion Biology, Department of Mathematical Sciences, Stellenbosch University, Matieland 7602, South Africa \\ ${ }^{\mathrm{d}}$ Mathematical and Physical Biosciences, African Institute for Mathematical Sciences, Muizenberg 7945, South Africa
}

\section{A R T I C L E I N F O}

\section{Keywords:}

Competitive hierarchy

Trade-off

Lyapunov exponent

Cellular automata

Spatial heterogeneity

\begin{abstract}
A B S T R A C T
Niche construction theory, which portrays organisms as active agents that modify their environment rather than mere passive entities selected by their environment, has received increasing attention in ecology and evolutionary biology. Here, we investigate the ecological consequences of niche construction in the system of three cyclically competing metapopulations, engaging a rock-scissors-paper game. Using cellular automata, we detected a variety of dynamic behaviors, including damped oscillation, periodical fluctuation and stage equilibrium, and the system transformed from disorder to order with gradually increasing niche-constructing intensity. Increasing niche-constructing intensity of a species, counterintuitively, reduced its own occupancy, but increased that of its inferior competitor. These species displayed interesting ripples in the two-dimension lattice space, with the pattern sensitive to the symmetry of competition intensity and other vital rates. Spatial heterogeneity induced by niche construction, together with the competition hierarchy, formed a stable and fixed range for each species with clear boundaries. Our results highlighted the necessity of investigating the adaptive dynamics of niche constructing traits to better understand the eco-evolutionary consequence of niche construction.
\end{abstract}

(c) 2016 Elsevier Inc. All rights reserved.

\section{Introduction}

Niche construction, where organisms can actively modify their environment rather than being passively selected by the environment, is a recent concept in evolutionary ecology [1-5]. It refers to the capacity of organisms to construct, modify, and select important components of their environment to meet their niche demand, such as through building nests and burrows, releasing chemicals and nutrients, which consequently modify both abiotic and biotic forces of natural selection in the local environment. In doing so, niche construction could generate a feedback between environmental processes and eco-evolutionary ones [4,6-10]. Niche construction could, arguably, be an evolutionary process by its own right, rather than a mere product of evolution [11-13]. Nonetheless, niche construction can be considered equivalent to ecosystem engineering at the ecological time scale, often through modifying the flow of energy and matters in ecosystems [2,3,14].

\footnotetext{
* Corresponding author at: School of Applied Mathematics, Guangdong University of Technology, No. 161, Yinglong Road, Tianhe District, Guangzhou 510520, PR China. Tel.: +86 1392510 5860; fax: +86 2087083272.

E-mail addresses: hanxzh@gdut.edu.cn (X. Han), sunnycby@126.com (B. Chen), chui@sun.ac.za (C. Hui).
} 
To date, the majority of theoretical models for describing the dynamics of niche construction have confirmed that niche construction can remarkably affect the dynamics and distribution of involved species $[2,3,8-10,15,16]$. For example, Hui et al. [15] found that organism-environmental feedbacks from niche construction can profoundly affect the outcome of competition and the maintenance of biodiversity in metapopulations, mainly through forming self-organized environmental heterogeneity and range-limited species distributions. Han and Zhang [16] expanded on Tilman's multi-species competition model by incorporating niche construction component to illustrate that niche construction also affects the ecological order of metapopulation, i.e. the sequence of populations' occupy proportion of habitat. In a model of two consumers that compete for one limiting resource but consumed by one common predator, niche construction can either generate net interspecific facilitation or strengthen interspecific competition via altering the balance between intraspecific and interspecific competitive effects [3]. Through rescuing the depleting resource, niche construction can also foster species coexistence [3]. Various processes of niche construction can also lead to diverse spatial distributions of species such as spiral wave, spiralbroken wave and circular wave [9], and it can even restrain gene flows along environmental gradients via forming fitness valleys [10].

Cyclic population dynamics, as manifested in the rock-scissors-paper game, have been previously studied by many for elucidating the mechanisms of biodiversity and self-organized patterns using lattice models and partial differential equations (e.g., [17-38]). Results from most these models show the emergence of self-organized spatial distribution of species and complex patterns of coexistence, such as entangled rotating spiral waves (e.g. [17,18,22-24,35,38]), remarkable targetwave $[25,38]$, chaotic patches [26] and phase transition of suddenly appearing large-scale fluctuations [20,36]. These various outcomes can be explained by the topological shape, spatial structure, interaction range and rate, as well as species (or player) numbers in the ecological or evolutionary network [30]. Reichenbach et al. [23] have analyzed the spiral waves from low mobility and computed the wavelength and spreading velocity of spirals. Evidently, biodiversity in a community experiencing cyclic competition depends heavily on the difference between species' mobility (or invasion rate) [26,30]. Protection spillovers where species $A$ indirectly facilitates species $B$ via suppressing the natural enemies of $B$, may also change the dynamics of cyclic dominance fundamentally in structured populations, which could underpin the phenomenon of microbial coexistence [38]. A recent study on the population dynamics of three cyclically competing species in a two-dimensional turbulent fluid system sheds new insight to how turbulent transportation affects ecosystem structure in communities without a clear competition hierarchy [32]. It has also been widely observed in cyclic game models of the dominance of inferior species (e.g., [17-19]). Studies on an extension of the rock-paper-scissors game, named the finger game [27] or rock-paperscissors-lizard-Spock (RPSLS) game [29,36] which includes five species along a cyclic hierarchy, also confirmed that a large number of species and high mobility can jeopardize biodiversity maintenance and potentially lead to diverging fluctuations under strong mixing [36]. In a six-species predator-prey cyclic system, an unexpected non-monotonous dependence of alliance survival on special heterogeneous invasion rates, even with the Gaussian noise, was revealed [33,34]. The extinction time of a well-mixed four cyclically competing species was estimated to have correlated linearly with the system size, and the probability distribution of extinction time takes roughly the shape of a shifted exponential distribution [28,31]. Szolnoki et al. [37] showed that the transition from pairwise to group interactions can decelerate or even reverse the direction of invasion between competing players. Time-dependent factors were also found important for cyclic system; different capacities of learning by players in the spatial prisoner's dilemma game offer alternative ways for the persistence of cooperators [35].

Cyclic competition can also emerge spontaneously in evolutionary public goods games with volunteers [39], peer punishment [40], pool punishment [41,42], reward [43,44] and reciprocity [45]. A spatial public goods game generally includes three elementary strategies: to defect, to reward and to punish. Other deformations of this classic setup have resulted in rich dynamic behaviors $[41,42,45]$. For example, defection remains viable when the rewarding is costly. Moderate rewards may promote cooperation better than big rewards, especially if the return from cooperative contributions is low [44]. Moreover, regardless of the return ratio which governs the public goods game, punishment in particular are more effective in deterring defection than the combined strategy of punishment, reward or defection [45]. Indeed, this topic has received a comprehensive review recently (see [30]).

Symmetry, meaning uniformity or invariance in patterns or structures, is often appealing and inspiring in natural systems [46]. Symmetry breaking is the process by which the uniformity is broken so to generate a more structured and improbable state in the focal system [47]. In general, increasing levels of broken symmetry in systems are often associated with increasing complexity and functional specialization of system components [46], and are true in biology across all system scales, from the macromolecules https://en.wikipedia.org/wiki/Symmetry_breaking_and_cortical_rotation-cite_note-3 to tissues and organs (see [48-55]). In ecological systems, symmetry breaking has received wide attention especially under the framework of cyclic competition, partly because the asymmetric scenarios can be diverse. For instance, if the dispersal rates of all species are close/similar to each other, the coexistence takes the form of spiral waves or target waves [22]. The spiral dynamics, however, do not appear if the species have unequal mobility. The greater the disparity between dispersal rates, the shorter the distance to the origin at which the spiral waves break down, and the dynamics begin to resemble several tangled spirals, eventually without forming spirals [26,27]. In systems of marine phytoplankton [56] and atmospheric microbes [57], advection and diffusive transportation in cyclic competition can further interact with the environment and form a complex interplay of diverse factors dictating symmetry breaking [32].

Here, we investigate the ecological consequences of niche construction in three cyclically competing species. Competition among these three species resembles the game of rock-scissors-paper (as in Zhang et al. [58]). The rock species $R$ can invade the habitat patches occupied by the scissors species $S$; the scissors species $S$ can invade the habitat patches occupied 
by the paper species $P$; and the paper species $P$ can invade the habitat patches occupied by the rock species $R$. There are a number of natural systems resembling our model setup, such as coral reef invertebrates [59], lizards in the inner coast range of California [60] and microbial populations of colicinogenic E. coli [61]. Based on the cyclic-competition model, we further allow the three species, through niche construction, to modify their own habitat resources and constrain the accessibility of resources of other species. By comparing the spatiotemporal dynamics from our model with those observed in Zhang et al. [58], we explore how niche construction affects the outcome and dynamics of cyclic competition, and how it interferes the effect of inter-species differences in vital rates on the emerged spatial patterns.

\section{Model}

Tilman's multi-species model of competing metapopulations assumes that all species are arranged in a simple competitive hierarchy, ranking species from the best competitor to the worst [62]. Superior competitors can invade habitat patches occupied by inferior competitors and displace them instantaneously, whilst inferior competitors cannot displace species of higher competitive hierarchies. Based on this competition model, Zhang et al. [58] constructed the following model for the rock (species 1 ), scissors (species 2) and paper (species 3) species engaging cyclic competition:

$$
\begin{aligned}
& \frac{d P_{1}}{d t}=c_{1} P_{1}\left(1-P_{1}-P_{3}\right)-e_{1} P_{1}-c_{3} P_{3} P_{1}, \\
& \frac{d P_{2}}{d t}=c_{2} P_{2}\left(1-P_{2}-P_{1}\right)-e_{2} P_{2}-c_{1} P_{1} P_{2}, \\
& \frac{d P_{3}}{d t}=c_{3} P_{3}\left(1-P_{3}-P_{2}\right)-e_{3} P_{3}-c_{2} P_{2} P_{3}
\end{aligned}
$$

where $P_{k}$ is the fraction of habitat patches occupied by species $k(=1,2,3) ; c_{k}$ and $e_{k}\left(c_{k}>e_{k}\right)$ are the colonization and extinction rate of species $k$, respectively.

We focus on examining the process of niche construction in these species. Let $R_{k}$ be the resource type needed by species $k$. Following Laland et al. [1,63], we define positive niche construction as a process that can increase the resource content. Specifically, we assume that through niche construction process a species can increase the amount of its own resource and reduce the amount of resource on which its inferior competitor relies. Further considering the processes of resource recovery and dissipation, we have the following resource dynamics equations,

$$
\begin{aligned}
& \frac{d R_{1}}{d t}=\alpha_{1} P_{1}-\alpha_{3} P_{3}-\gamma_{1} R_{1}+\varepsilon_{1}, \\
& \frac{d R_{2}}{d t}=\alpha_{2} P_{2}-\alpha_{1} P_{1}-\gamma_{2} R_{2}+\varepsilon_{2}, \\
& \frac{d R_{3}}{d t}=\alpha_{3} P_{3}-\alpha_{2} P_{2}-\gamma_{3} R_{3}+\varepsilon_{3}
\end{aligned}
$$

where $\alpha_{k}, \gamma_{k}$ and $\varepsilon_{k}(k=1,2,3)$ are respectively the intensity of niche construction, the independent process of resource dissipation and recovery, satisfying the condition that $\alpha_{k}, \gamma_{k}$ and $\varepsilon_{k}$ lie within the interval $[0,1]$ and that $0 \leq \alpha_{k}+\gamma_{k}+\varepsilon_{k} \leq$ 1. If there is no niche construction $\left(\alpha_{k}=0\right)$, the equilibrium of resource $k$ is given by $R_{k 0}=\varepsilon_{k} / \gamma_{k}$. For simplicity, we fix the values of $\gamma_{k}(=0.2)$ and $\varepsilon_{k}(=0.1)$ in the following analyses but only focus on the role of changing the intensity of niche construction.

The ability for effectively selecting feeding sites is a major determinant of species' survival, growth, and reproductive success. Some evidence indicates that cost-benefit relationships (e.g., resource availability and competition risks) may affect the behavior of animals [64,65]. Optimal foraging theory predicts that animals should forage for longer periods in high-quality patches than in poor-quality ones [66]. The spatial distribution of resources is particularly important for determining competition outcomes. To depict the effect of niche construction more intuitively, we define the colonization rate of a species as an increasing function with resource suitability. Using the simplest form of resource utilization spectrum, we assume that the optimum resource level of a species is equal to the equilibrium of resource level without niche construction, $R_{k 0}$, and hence the resource suitability of each species can be defined as $\omega_{k}=\exp \left[-\lambda_{k}\left(R_{k}-R_{k 0}\right)^{2}\right]$, where $1 / \lambda_{k}$ depicts the niche breadth of species $k$. In the following, we let $\lambda_{k}=3$. The colonization rate of species $k$ is assumed to be proportional to the resource suitability, $c_{k}=c_{k 0} \omega_{k}$, where $c_{k 0}(k=1,2,3)$ is the maximum colonization rate. Furthermore, as there is only a limited amount of time and energy for growth, structure maintenance and reproduction, an organism must allocate its captured resources among these alternative needs [67]. To this end, we consider a simple trade-off between the maximum colonization rate and the ability of niche construction, $c_{k 0}+\alpha_{k}=1$.

To investigate the spatial dynamics and pattern formation of niche-constructing metapopulations, we construct a cellular automaton in a 2D lattice arena with a number of $n \times n$ patches with synchronized updating, von Neumann neighbors and periodic boundaries. Simulations were implemented according to the probability transition model $[68,69]$. Assuming $P_{k, t}(i, j)$ indicates the probability that patch $(i, j)$ is occupied by a local population of species $k$ at time $t$, we have the following 
probability transition rules:

$$
\begin{aligned}
& P_{1, t+1}(i, j)=P_{1, t}(i, j)+\left[c_{10} \omega_{1, t}(i, j) \frac{\sum P_{1, t}(i, j)}{4}\left(1-P_{1, t}(i, j)-P_{3, t}(i, j)\right)-e_{1} P_{1, t}(i, j)\right]-c_{30} P_{3, t}(i, j) P_{1, t}(i, j) \\
& P_{2, t+1}(i, j)=P_{2, t}(i, j)+\left[c_{20} \omega_{2, t}(i, j) \frac{\sum P_{2, t}(i, j)}{4}\left(1-P_{1, t}(i, j)-P_{2, t}(i, j)\right)-e_{2} P_{2, t}(i, j)\right]-c_{10} P_{1, t}(i, j) P_{2, t}(i, j) \\
& P_{3, t+1}(i, j)=P_{3, t}(i, j)+\left[c_{30} \omega_{3, t}(i, j) \frac{\sum P_{3, t}(i, j)}{4}\left(1-P_{2, t}(i, j)-P_{3, t}(i, j)\right)-e_{3} P_{2, t}(i, j)\right]-c_{20} P_{2, t}(i, j) P_{3, t}(i, j)
\end{aligned}
$$

where $\sum P_{k, t}(i, j)$ is the sum of the probabilities of species $k$ in the four neighboring patches at time $t$. Correspondingly, the dynamics of different resources (only giving the case of $R_{2}$ for short) in patch $(i, j)$ is given by:

$$
R_{2, t+1}(i, j)=R_{2, t}(i, j)+\left[\alpha_{2} P_{2, t}(i, j)-\alpha_{1} P_{1, t}(i, j)-\gamma_{2} R_{2, t}(i, j)+\varepsilon_{2}\right]
$$

Note that we did not implement the possibility of resource diffusion between patches as it is normally much slower than the movement of individuals. Using the probability transition model, the spatial distribution patterns of cyclic competition can be explicitly revealed.

\section{Results}

Let us consider a simple case of symmetric (equal) vital rates among species $\left(c_{k}=c, \alpha_{k}=\alpha\right.$ and $e_{k}=e$ ); that is, the three species are identical in their ecological properties except along a cyclic competition hierarchy. Setting the right side of Eq. (1) to zero, we can obtain a nontrivial equilibrium of the system:

$$
P^{*}=\left(P_{1}^{*}, P_{2}^{*}, P_{3}^{*}\right)=\left(\frac{1-\frac{e}{c}}{3}, \frac{1-\frac{e}{c}}{3}, \frac{1-\frac{e}{c}}{3}\right)
$$

Because of $c+\alpha=1$, the nontrivial equilibrium can also be expressed as $\frac{1}{3}\left(1-\frac{e}{1-\alpha}, 1-\frac{e}{1-\alpha}, 1-\frac{e}{1-\alpha}\right)$, which needs to satisfy the condition $\alpha+e<1$ to ensure the metapopulation persistence.

For an asymmetrical structure that the three species are distinct in their colonization, extinction and niche constructing rates, more complicated equilibriums and various dynamical behaviors emerge. The non-trivial equilibrium of the metapopulations is as follows:

$$
\begin{aligned}
& P_{1}^{*}=\frac{1-\frac{e_{1}}{c_{1}}-\left(1-\frac{e_{3}}{c_{3}}\right) l_{3}+\left(1-\frac{e_{2}}{c_{2}}\right) l_{2} l_{3}}{1+l_{1} l_{2} l_{3}} \\
& P_{2}^{*}=\frac{1-\frac{e_{2}}{c_{2}}-\left(1-\frac{e_{1}}{c_{1}}\right) l_{1}+\left(1-\frac{e_{3}}{c_{3}}\right) l_{3} l_{1}}{1+l_{1} l_{2} l_{3}} \\
& P_{3}^{*}=\frac{1-\frac{e_{3}}{c_{3}}-\left(1-\frac{e_{2}}{c_{2}}\right) l_{2}+\left(1-\frac{e_{1}}{c_{1}}\right) l_{1} l_{2}}{1+l_{1} l_{2} l_{3}}
\end{aligned}
$$

where $l_{1}=1+\frac{c_{1}}{c_{2}}, l_{2}=1+\frac{c_{2}}{c_{3}}, l_{3}=1+\frac{c_{3}}{c_{1}}$. The equilibrium of metapopulations not only depends on the original occupancy equilibrium $\left(1-e_{i} / c_{i}\right)$, but also on the ratio of a species' colonization rate to that of its inferior competitor $\left(l_{i}\right)$. Meanwhile, the equilibrium of resources is given as the following:

$$
\begin{aligned}
& R_{1}^{*}=\frac{\varepsilon_{1}}{\gamma_{1}}+\frac{P_{1}^{*} \alpha_{1}-P_{3}^{*} \alpha_{3}}{\gamma_{1}} \\
& R_{2}^{*}=\frac{\varepsilon_{2}}{\gamma_{2}}+\frac{P_{2}^{*} \alpha_{2}-P_{1}^{*} \alpha_{1}}{\gamma_{2}} \\
& R_{3}^{*}=\frac{\varepsilon_{3}}{\gamma_{3}}+\frac{P_{3}^{*} \alpha_{3}-P_{2}^{*} \alpha_{2}}{\gamma_{3}}
\end{aligned}
$$

Clearly, the resource equilibrium is not only affected by the processes of resource dissipation and recovery, but also by the niche-constructing behavior, implying the importance of niche construction for the resource level and thus the resource suitability and persistence of each species.

When fixing parameters $\varepsilon_{k}$ and $\gamma_{k}$, the system dynamics is mainly affected by the parameters of niche-constructing intensity $\left(\alpha_{k}\right)$ (thus also the colonization rate through the trade-off) and extinction rate $\left(e_{k}\right)$ of species $k(=1,2,3)$. We first observed the dynamical behaviors of the system using numerical simulations. Parameters were chosen to reflect the symmetry and various hierarchies of the system so that all possible outcomes can be embodied. From the diagrams of occupancy frequencies and trajectories in the phase space (shown in Fig. 1), three typical oscillatory dynamics were presented, including the stage equilibrium (Fig. 1a-d), the periodical oscillation of limit cycle (Fig. 1e and f), the damped oscillation (Fig. $1 \mathrm{~g}-\mathrm{j}$ ). When the three species were symmetrical (identical in vital rates), the amplitude of their oscillatory dynamics increased synchronously and stayed at an equal level (Fig. 1a and b). However, this synchronized and identical dynamics 
a

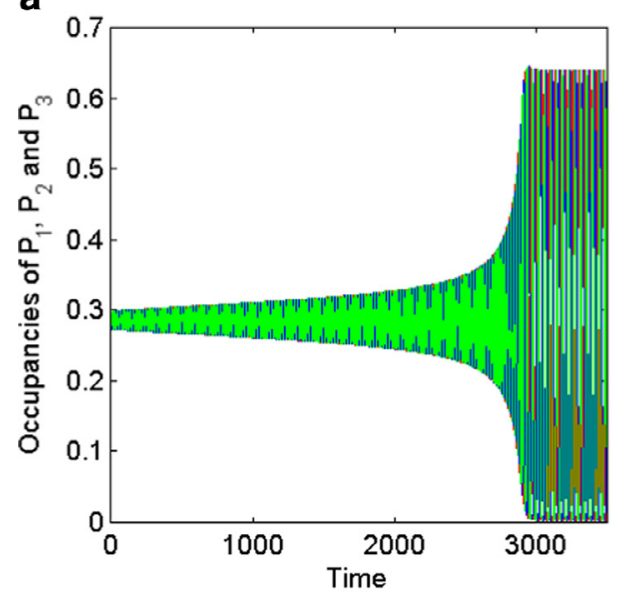

C
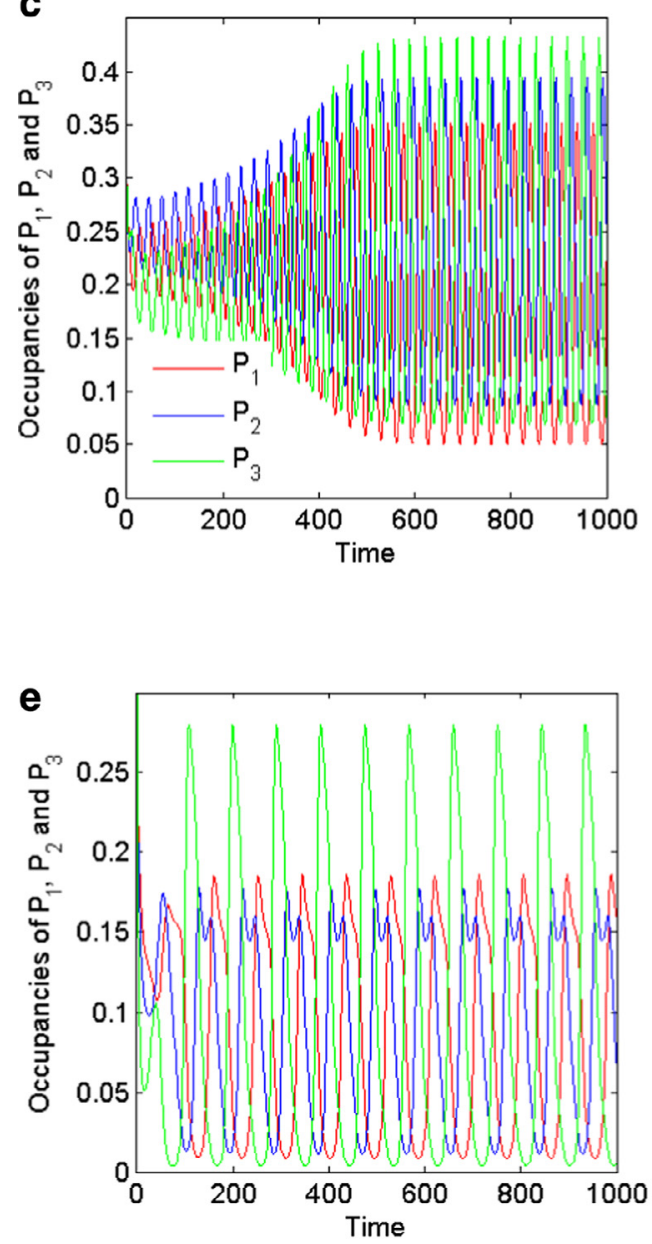

b

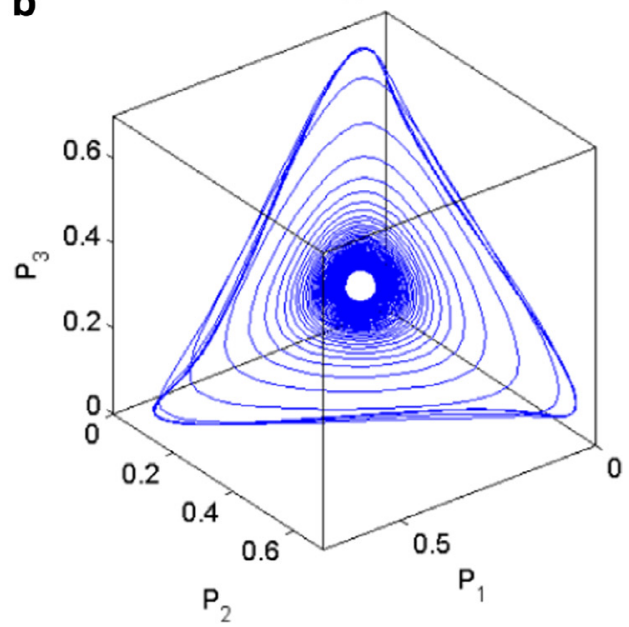

d
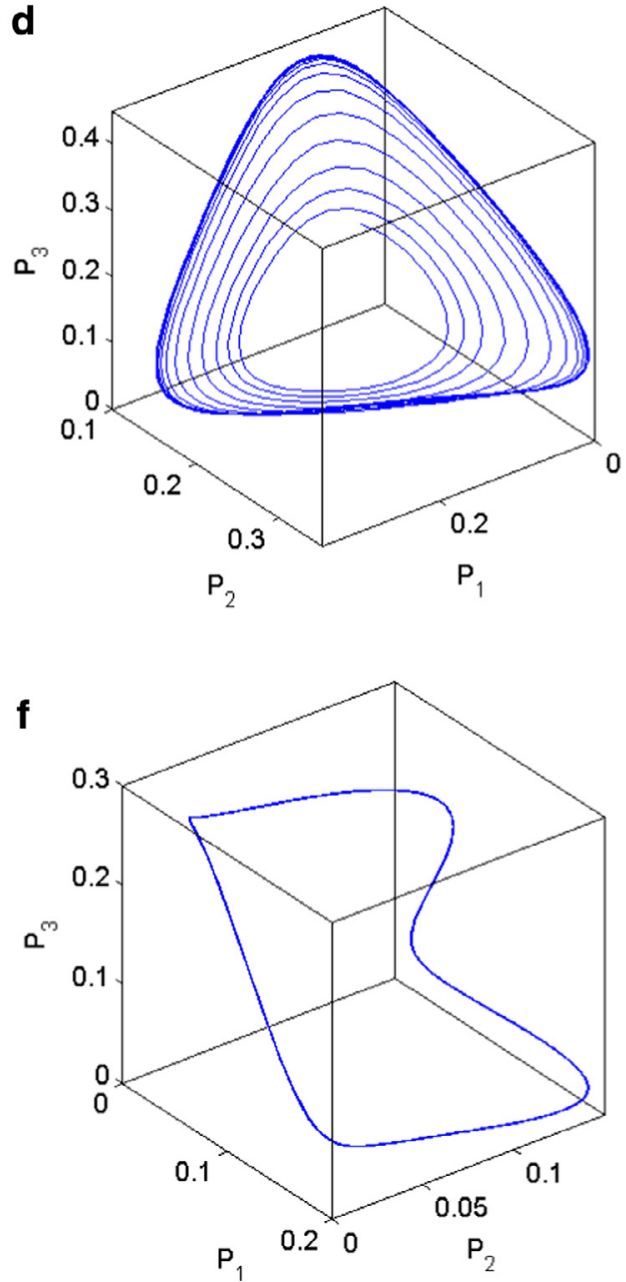

Fig. 1. Temporal dynamics and phase-plane portraits of occupancies of the three species. Parameters are (a) and (b) $\alpha_{1}=\alpha_{2}=\alpha_{3}=0.3, e_{1}=e_{2}=e_{3}=$ $0.1 ;$ (c) and (d) $\alpha_{1}=0.5, \quad \alpha_{2}=0.4, \quad \alpha_{3}=0.3, \quad e_{1}=0.1, \quad e_{2}=e_{3}=0.2 ;$ (e) and (f) $\alpha_{1}=0.7, \quad \alpha_{2}=0.5, \quad \alpha_{3}=0.2, \quad e_{1}=0.1, \quad e_{2}=0.3, \quad e_{3}=0.5 ;$ (g) and (h) $\alpha_{1}=0.5, \quad \alpha_{2}=0.4, \quad \alpha_{3}=0.4, \quad e_{1}=0.1, \quad e_{2}=e_{3}=0.2$; (i) and (j) $\alpha_{1}=\alpha_{2}=\alpha_{3}=0.2, \quad e_{1}=0.2, \quad e_{2}=0.1, \quad e_{3}=0.05$. The red, blue and green colors respectively refer to species $P_{1}, P_{2}$ and $P_{3}$. (For interpretation of the references to color in this figure legend, the reader is referred to the web version of this article.) 

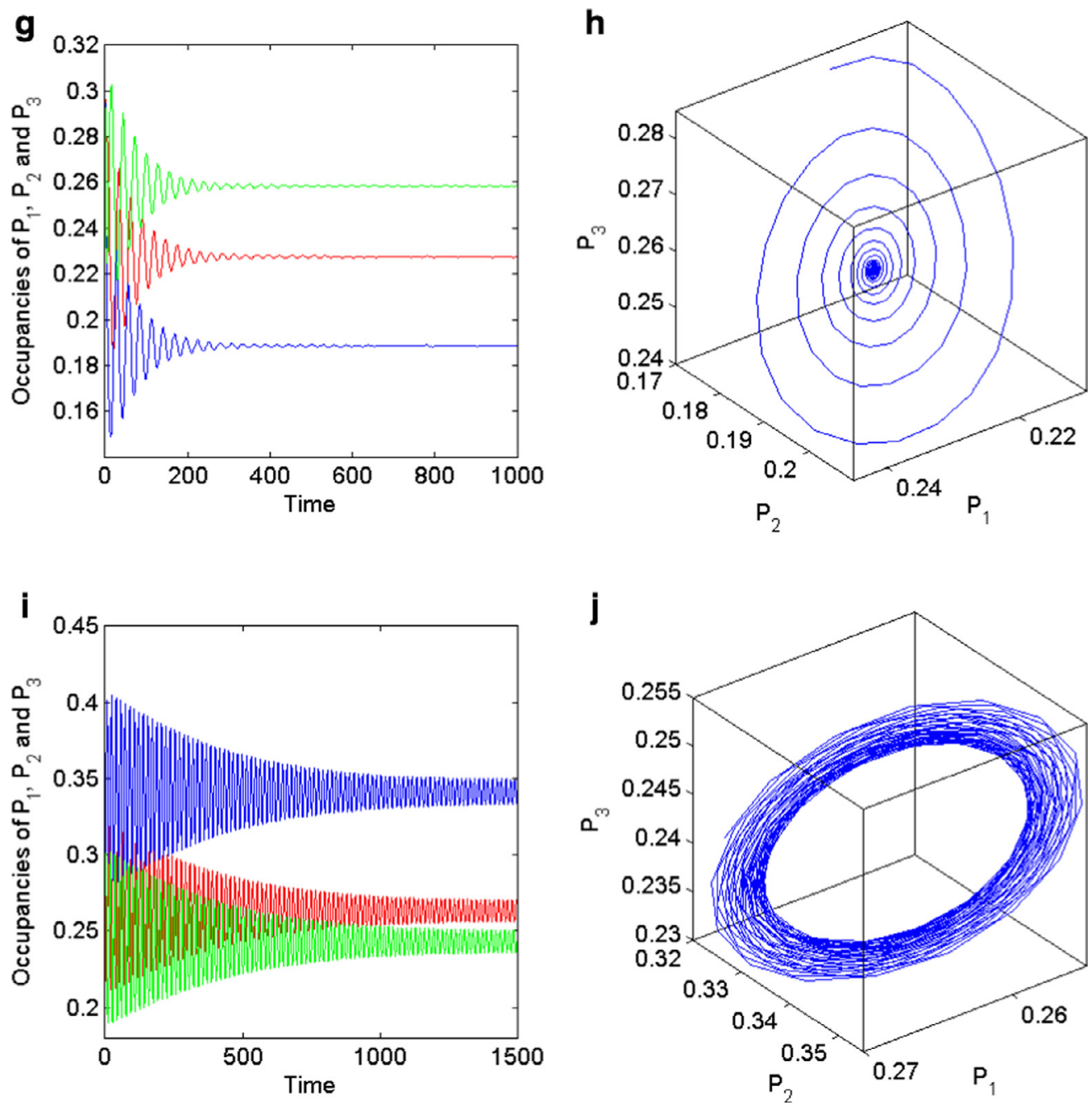

Fig. 1. Continued

broke once allowing differences in species' niche constructing intensity. Interestingly, high intensity of niche construction always led to a low level of occupancy (Fig. 1c and d). When the three niche-constructing intensities were set along a hierarchy $\left(\alpha_{1}>\alpha_{2}>\alpha_{3}\right)$, the system exhibited the limit cycle (Fig. 1e and f). The above dynamics of the system are the typical, representing all possible behaviors in the cyclic networks, which have been referred to as oscillatory, absorbing and stationary phases (sensu [30]).

Bifurcation analysis was conducted to demonstrate the effect of changing niche-constructing intensity on metapopulation dynamics. Maximal conditional Lyapunov exponent was calculated for checking the system sensitivity to the initial values of state variables and whether chaos behavior exists. Fixing three extinction rates as $e_{i}=0.1(\mathrm{i}=1,2,3)$ and assuming that the niche-constructing intensities of species 1 and 3 are equal. As the increasing of $\alpha_{1}\left(=\alpha_{3}\right)$, we examined how the changing niche-constructing intensity of species 2 affects system dynamics (Fig. 2). Two obvious features appeared when $\alpha_{2}$ increases. First, the Lyapunov exponents of the system are $(0,0,-)$ at the point of $\alpha_{1}=\alpha_{2}=\alpha_{3}$ and its neighborhood, which means the torus of system. When $\alpha_{2}>0.5$, the Lyapunov exponents are $(-,-,-)$. Here, species 1 was nearly extinct, and the system converged to a fixed point. Moreover, species 2 exhibited marked advantages in occupancy when $\alpha_{2}<\alpha_{1}=\alpha_{3}$, but gradually became the inferior when $\alpha_{2}>\alpha_{1}=\alpha_{3}$. Species 1 and 3 , meanwhile, were converging to opposite directions, with species 3 eventually becoming dominated in number. This result suggests that the increase of niche-constructing intensity of a given species can counterintuitively decrease its own occupancy but increase the occupancy of its inferior competitor (Fig. 2a and c). The situation of $\alpha_{1}=\alpha_{2}=\alpha_{3}$ is an important turning point of system dynamics. The system transformed from disorder (Fig. 2b) to more orderly behavior (Fig. 2h) with gradually increasing niche-constructing intensities of species 1 and $3\left(\alpha_{1}=\alpha_{3}\right)$.

As asymmetrical conditions produced more complex behavior, we further examined the effects of inter-species difference on system behavior. Let $\alpha_{1}=\alpha_{2}-\delta$ and $\alpha_{3}=\alpha_{2}+\delta$, where $\delta$ is the parameter that describe inter-species difference in 


\section{a}

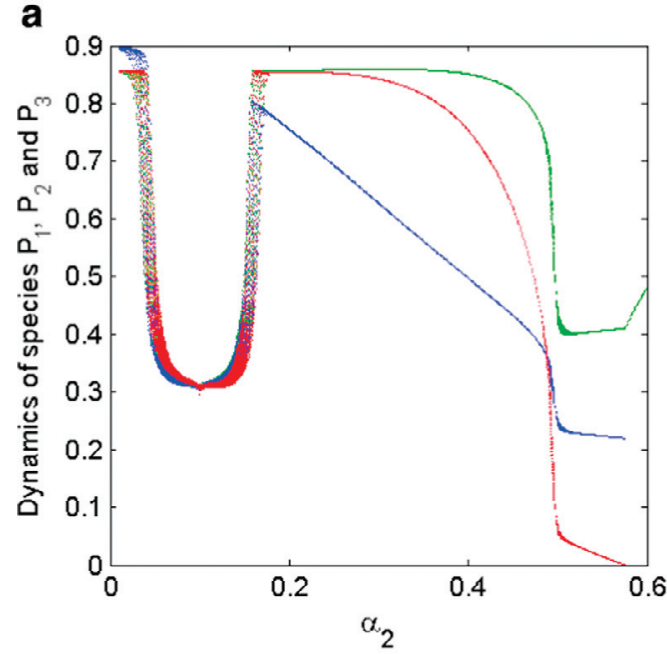

C

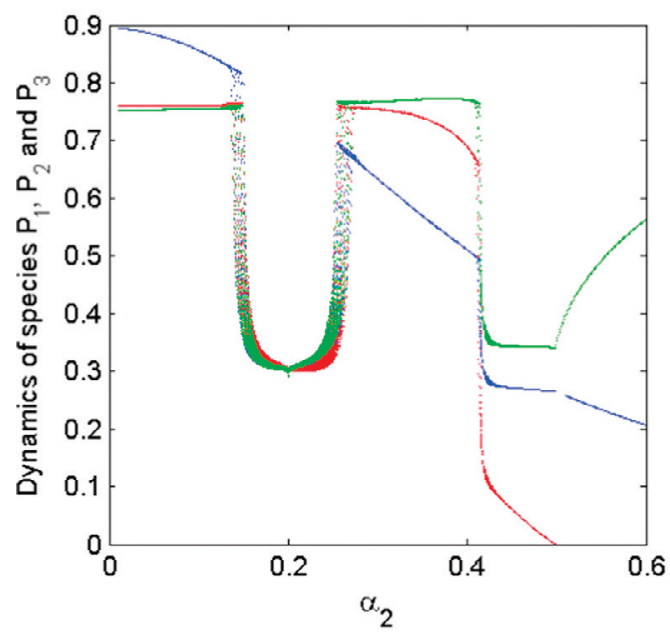

e

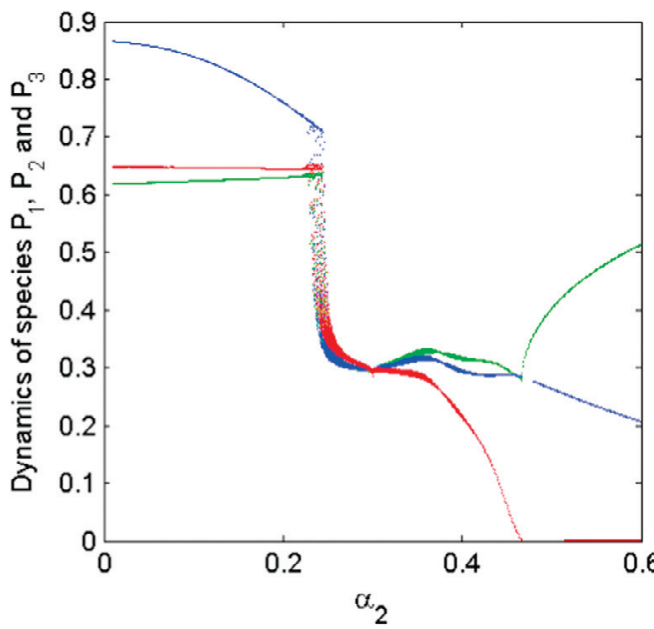

b

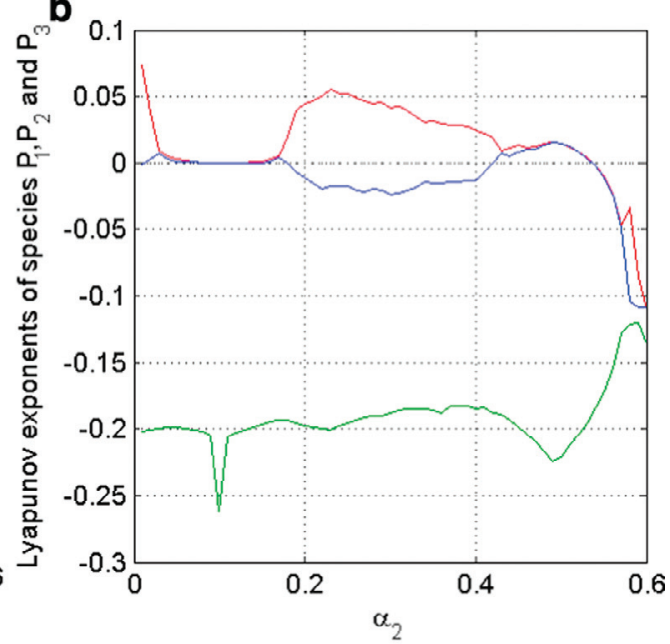

d

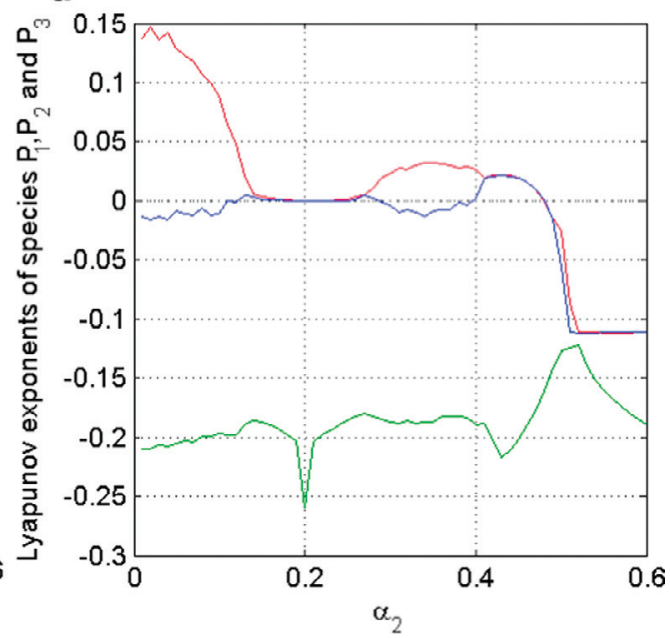

f

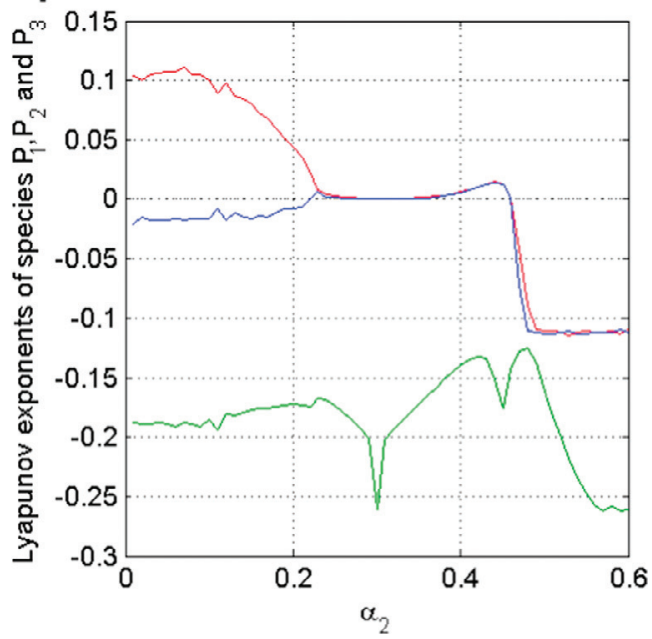

Fig. 2. The bifurcation diagram and corresponding Lyapunov exponents of three species as a function of increasing niche-constructing intensity. Parameters are $\alpha_{1}=\alpha_{3}=0.1$ in (a) and (b); $\alpha_{1}=\alpha_{3}=0.2$ in (c) and (d); $\alpha_{1}=\alpha_{3}=0.3$ in (e) and (f); $\alpha_{1}=\alpha_{3}=0.4$ in (g) and (h). The extinction rates are $e_{1}=e_{2}=$ $e_{3}=0.1$. The red, blue and green lines represent the species 1,2 and 3 . (For interpretation of the references to color in this figure legend, the reader is referred to the web version of this article.) 

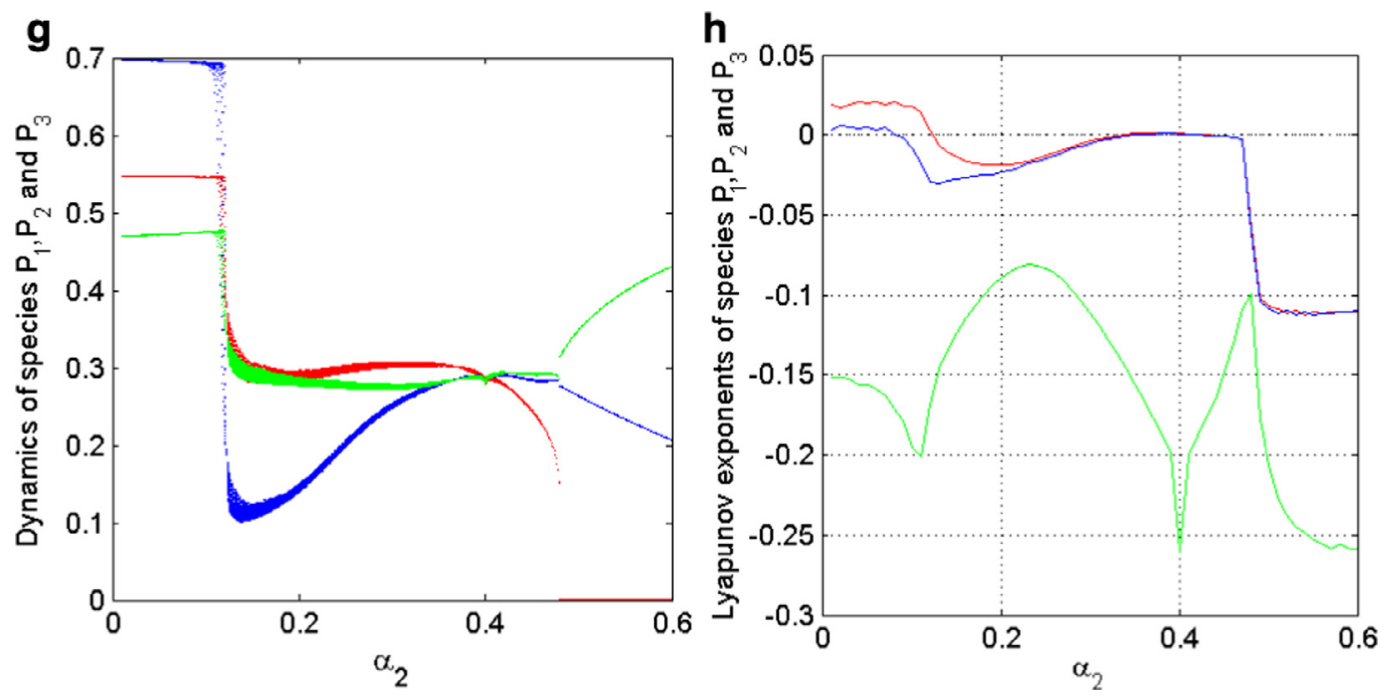

Fig. 2. Continued

a

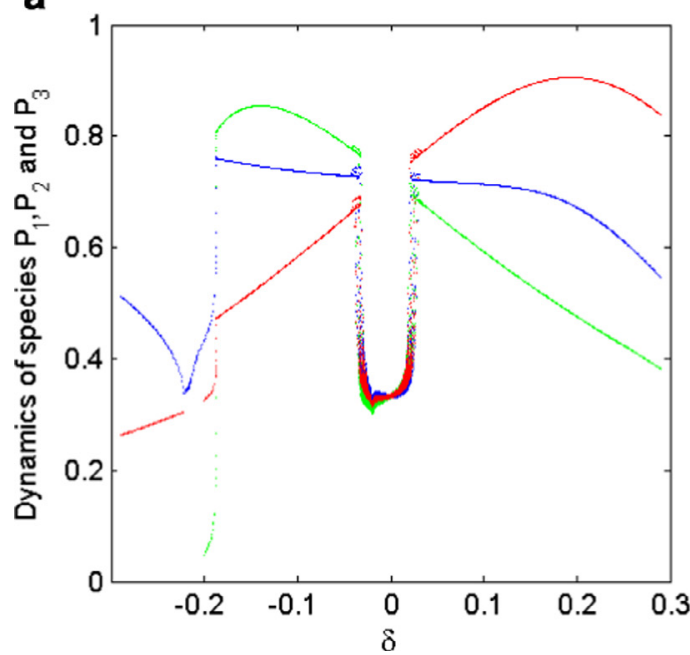

b

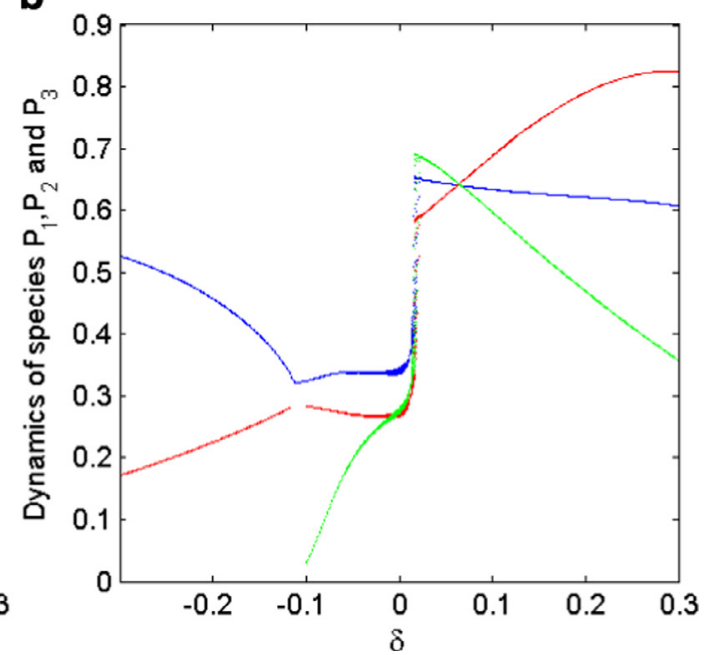

Fig. 3. The bifurcation diagrams of three species with increasing difference in niche-constructing intensities among species $\delta$. We keep $\alpha_{2}=0.3$; (a) extinction rates of three species are equal: $e_{1}=e_{2}=e_{3}=0.05$; (b) extinction rates are: $e_{1}=0.15, e_{2}=0.1, e_{3}=0.05$. The red, blue and green lines represents the species 1, 2 and 3. (For interpretation of the references to color in this figure legend, the reader is referred to the web version of this article.)

niche-constructing intensity. Fixing the value of parameter $\alpha_{2}$, we thus have that, the larger parameter $\delta$ is, the greater the differences among species are. Moreover, the condition $\alpha_{1}>\alpha_{2}>\alpha_{3}$ is satisfied when $\delta<0$, while $\alpha_{1}\left\langle\alpha_{2}<\alpha_{3}\right.$ when $\left.\delta\right\rangle 0$. Bifurcation diagrams of the three species influenced by $\delta$ were drawn (Fig. 3 ). Under the condition of equal extinction rates of the three species (Fig. 3a), the occupancies of these species moved in opposite directions with their niche-constructing intensities, excepting $\delta \in(-0.03,0.03)$. More specifically, we have $\mathrm{P}_{1}<P_{2}<P_{3}$ when $\delta \in(-0.2,-0.03)$, while $\mathrm{P}_{1}>P_{2}>P_{3}$ when $\delta \in(0.03,0.3)$. Moreover, the bigger the absolute value of parameter $\delta$ was, the more the difference of three species' occupancies were. For the situation of unequal extinction rates (Fig. 3b), the overall trend is similar to Fig. 3a. Although the extinction rate of species 3 was minimal, its survival range was the narrowest. When $\delta<-0.1$, species 3 almost definitely cannot survive. Species 2 had a bigger occupancy than species 1, suggesting that the species with a lower colonization rate and a higher extinction rate cannot reverse the disadvantage of situation, even if it has a stronger niche-constructing ability. When $\delta>0$ (i.e. $\alpha_{1}\left\langle\alpha_{2}<\alpha_{3}, \quad c_{1}\right\rangle c_{2}>c_{3}$, and setting $\mathrm{e}_{1}>\mathrm{e}_{2}>e_{3}$ ), the occupancy of species 1 and 3 respectively went straight up and then suddenly dropped, while that of species 2 remained at the same level. These results suggest that the role of niche construction is heightened in the cases of either minor differences of niche construction among species or small magnitude of the difference between colonization rate and extinction rate of species (e.g. the species 3 when $\delta=0.3$ ). The ability of niche-construction cannot completely determine the fate of system, only partially playing the regulation role for cyclic competition. In addition, the threshold values leading to the extinction of species 3 were $\delta=-0.2$ in Fig. $3 a$ and 
a

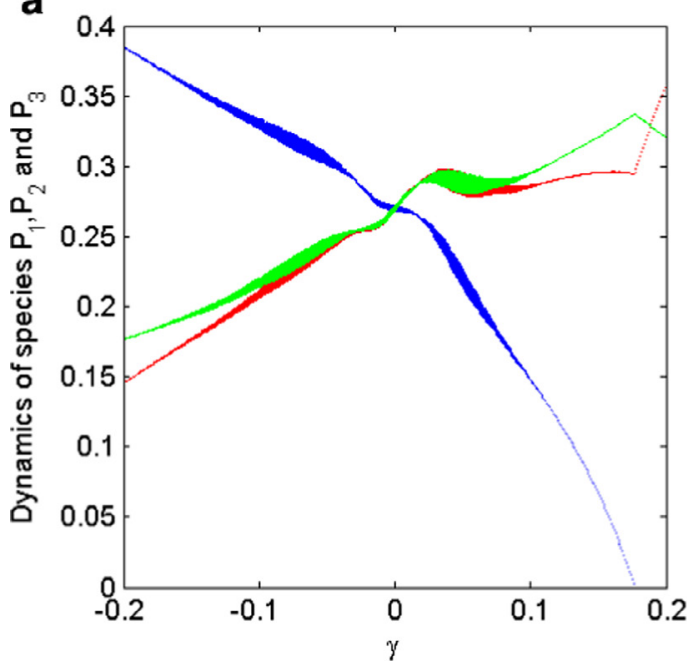

b

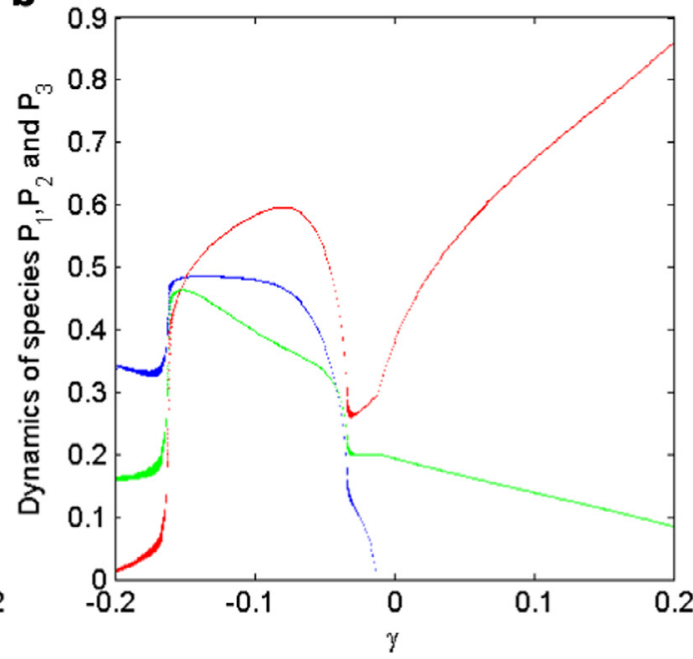

Fig. 4. The bifurcation diagrams of three species influenced by difference in extinction rate $\gamma$. We keep $e_{2}=0.2$, then $e_{1}=e_{2}-\gamma$, $e_{3}=e_{2}+\gamma ;$ the nicheconstructing intensities are (a) $\alpha_{1}=\alpha_{2}=\alpha_{3}=0.2$; (b) $\alpha_{1}=0.1, \alpha_{2}=0.3, \alpha_{3}=0.5$. The red, blue and green lines represents the species 1,2 and 3. (For interpretation of the references to color in this figure legend, the reader is referred to the web version of this article.)

$\delta=-0.1$ in Fig. 3b, respectively, because of the different initial values of three resources. It is also the reason of asymmetry showing between positive and negative parameters $\delta$.

Species dynamics was also affected by their extinction rates. Considering the trade-off between niche construction and colonization and the necessary condition of persistence $c_{k}>e_{k}$, we assumed $\alpha_{k}+e_{k}<1$ and selected two scenarios to discuss the effect of extinction rates on the dynamics of the three species. Also assume $\mathrm{e}_{1}=\mathrm{e}_{2}-\gamma$ and $\mathrm{e}_{3}=\mathrm{e}_{2}+\gamma$, where $\gamma$ is the parameter describing extinction rate's difference. The bifurcation diagrams were shown in Fig. 4a $\left(\alpha_{1}=\alpha_{2}=\alpha_{3}=0.2\right)$ and Fig. 4b $\left(\alpha_{1}=0.1, \alpha_{2}=0.3, \alpha_{3}=0.5\right)$. Obviously, when there was no difference in species' niche-constructing intensities, the system dynamics was completely determined by extinction rates. With parameter $\gamma$ increasing from negative to positive, the extinction rates of three species was gradually transformed from a descending trend to an increasing trend. During this change, the occupancy of species 2 sharply declined and even perished; in contrast occupancies of those other two species were rising steadily (Fig. 4a). In other words, the increase of extinction rate for a given species (species 3) can lead to the reduction of its superior competitor's occupancy, but counter-intuitively increase the occupancy of its own and its inferior competitor. Even if the extinction rate of species 3 was the highest, it may still have a high occupancy. This result suggests that the cyclic competitive structure of a system is a key factor to dictate the fates of the three species in case of equal niche-constructing intensities. The shape of the occupancy of these three species resembles the Greek letter $\Omega$ when the niche-constructing intensity differed among species $\left(\alpha_{1}<\alpha_{2}<\alpha_{3}\right.$, Fig. 4b). Different niche-constructing intensities of the three species pushed the extinction of competitive superior: species 2 became extinct when parameter $\gamma>0$. The most inferior niche constructor (species 1 ), however, jumped quickly to a higher level of occupancy. The shape of ' $\Omega$ ' emerged when $\gamma<0$, particularly in the interval $\gamma \in(-0.15$, 0$)$ when species occupancies satisfied $\mathrm{P}_{1}>\mathrm{P}_{2}>\mathrm{P}_{3}$. Overall, species 1 was the most sensitive one to the difference in extinction rate when niche construction differed among species.

The probability transition model with local dispersal between adjacent patches revealed complicated spatial patterns from the cyclic competition in niche-constructing metapopulations. We selected the arena of $200 \times 200$ lattices and presented snapshots of the three species when temporal dynamics of the system reached equilibrium after about 3000 time steps. There were two typical distribution patterns emerged, with the common one of ripples (Fig. 5a) and rare one of rings (Fig. 5b). The spatial pattern changed from tiny ripples to clumped rings with the increase of niche construction intensity. Furthermore, species 1 is always chasing after species 2, species 2 after species 3 and species 3 after species 1 . Such local chasing can form riffle-like spatial patterns. The result suggests that niche construction, to a certain degree, constrains the cyclic competition by the feedback between species and their habitat resources. The inter-species differences also have important roles on the spatial patterns of species. The boundary of species distribution, whether their extinction rates were equal or not, became more distinct with gradually increasing inter-species difference $\delta$ (shown in Fig. 6). Especially in Fig. 6b, the ring pattern only appeared at low level of inter-species difference $(\delta=0.05)$, and this pattern was very easy to be broken with only a slight increase of inter-species variation. It is worth noting that the spatial pattern does not monotonously depend on parameter $\delta$. Namely, the spatial correlation length decreases but then increases with the increase of $\delta$. Since the correlation length is considered a metric for quantifying spatial synchrony and aggregation [70,71], this result revealed a nonlinear effect of inter-species variation in resource renewing rate on the synchrony of spatial patterns. We suspect that this nonlinear response in spatial synchrony is related to the flipping phenomenon presented in Fig. 3b, where increasing 
a

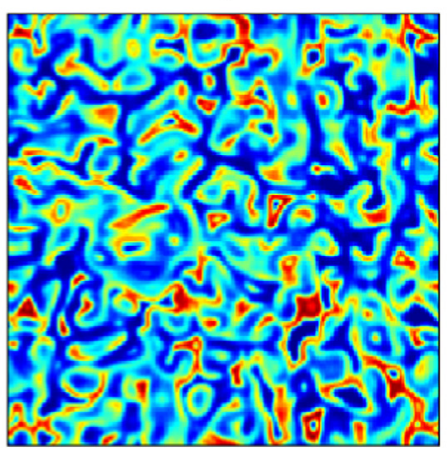

b

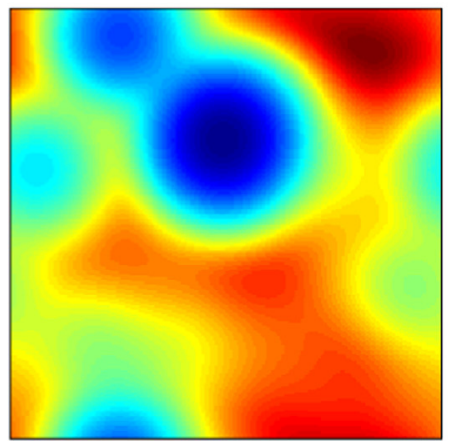

$P_{2}$
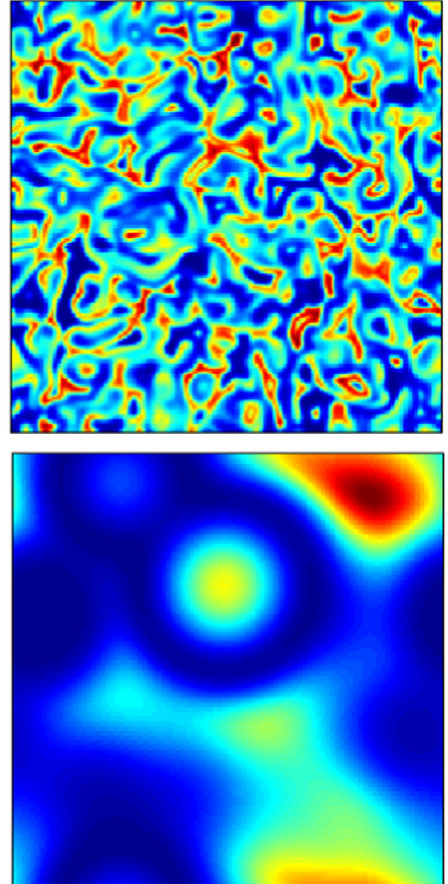

$\mathrm{P}_{3}$
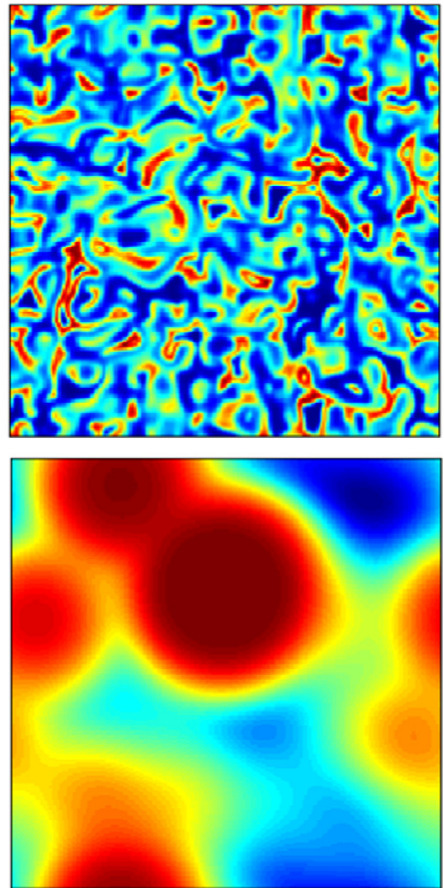

Fig. 5. The spatial patterns of three species with symmetric cyclic competition of niche-constructing metapopulations. The snapshots are at $t=3000$. Three species are with identical intensities of niche construction (a) $\alpha_{1}=\alpha_{2}=\alpha_{3}=0.2$ and (b) $\alpha_{1}=\alpha_{2}=\alpha_{3}=0.4$, extinction rates are $\mathrm{e}_{1}=\mathrm{e}_{2}=\mathrm{e}_{3}=0.1$.

a
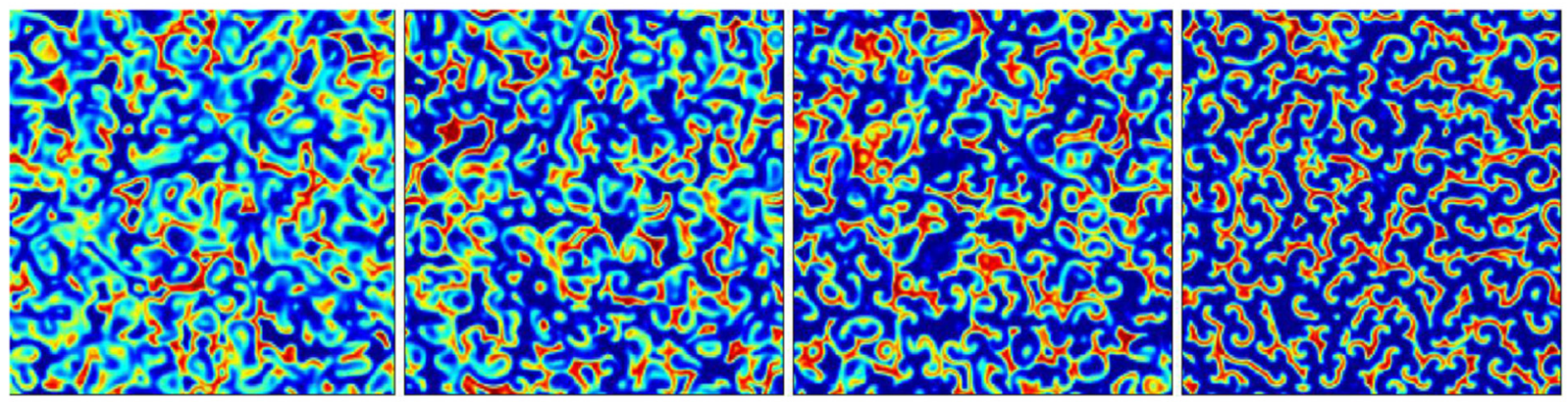

b
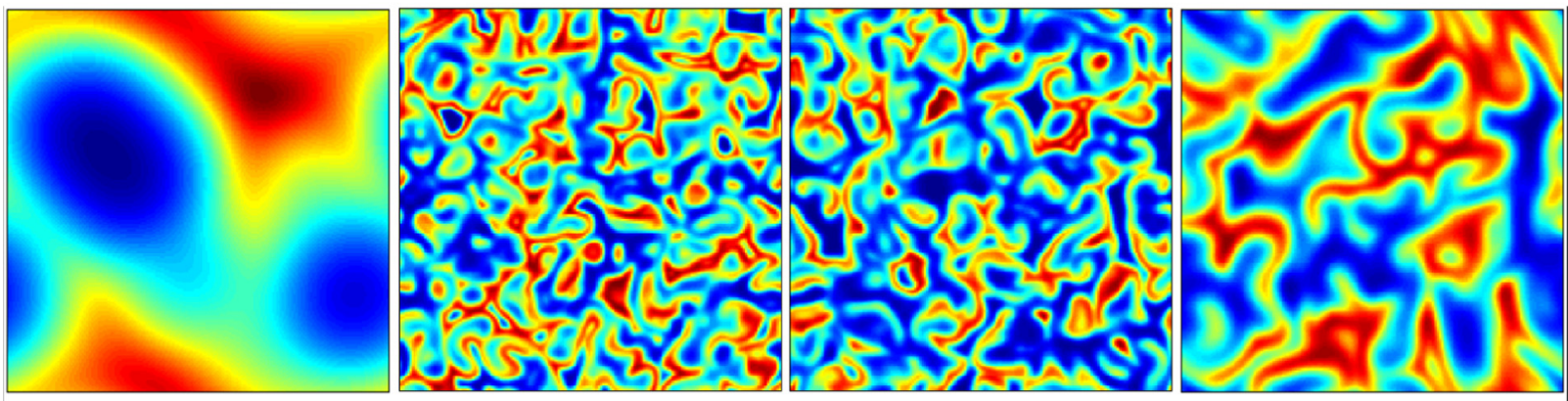

Fig. 6. The spatial patterns of species 2 under asymmetric cyclic competition. The colonization rate of each species satisfies the condition of $c_{i}=1-$ $\alpha_{i}(i=1,2,3)$. Assume parameter $\delta$ describes the interspecies difference, then the three species' abilities of niche construction are $\alpha_{1}=\alpha_{2}-\delta, \alpha_{2}$ and $\alpha_{3}=\alpha_{2}+\delta$. The snapshots are at $\mathrm{t}=3000$. (a) Parameter $\delta$ from left to right respectively is $0.05,0.1,0.15$ and 0.2 . Other parameters are $\alpha_{2}=0.2$ and $e_{1}=$ $e_{2}=e_{3}=0.1$; (b) parameters $\delta$ from left to right are 0.05, 0. 1, 0.2 and 0.3. Other parameters are $\alpha_{2}=0.3, e_{1}=0.25, e_{2}=0.2$, and $e_{3}=0.15$. 
difference in niche-constructing intensities first led to the reduction of occupancy difference but eventually the increase of occupancy difference after two species switched their dominance.

\section{Discussion}

We have examined the metapopulation model of three niche-constructing species engaging cyclic competition. Through numerical simulations and cellular automata, the system exhibited the dynamics of damped oscillation, periodical fluctuation and stage equilibrium, determined by the intensity of niche construction and extinction rates. In the two-dimensional arena, the three species displayed the spatial patterns of ripples and rings. Diversity in spatial patterns depends on the interspecies differences. The niche construction, which increases their own resource level but reduces the resource of its inferior competitor, plays an important role in system stability and supports species coexistence. Kylafis and Loreau [2] also indicated that niche construction can modify the traditional niche-deteriorating impacts of its agent or its competing species, and thus promotes the potential for species coexistence. Other studies have also supported this conclusion that niche construction can be a key factor for persistence and coexistence in competitive environment $[10,15,16,72]$.

Besides the spatial pattern of ripples, niche construction can also lead to ring-like spatial patterns; this latter one has not been observed in pure cyclically competing metapopulations [58], but was observed in time-lagged niche constructing metapopulations [9]. High intensity of niche construction is likely contributing to the formation of spatial rings. In the metapopulation framework, it has been proved that niche construction is a significant cause of spatial heterogeneity in the environment, through self-regulating feedbacks between organisms and their environment [9,15,72]. The spatial pattern of rings, nevertheless, can be easily disrupted by even small difference in niche construction intensity (Fig. 6b). With the increase of difference among niche-constructing intensities, regardless of the spatial patterns, species distribution became clearly static and fixed in space, distinctively demarcating the boundary of each species. Therefore, niche construction can play the role of a bridge between spatial heterogeneity and biotic interactions to dictate environmental heterogeneity and species' distribution limit $[10,15]$.

The trade-off between the intensity of niche construction and the colonization rate assumed in our model is essential for exploring the system dynamics. Charnov [73] emphasized that the evolutionary theory of life-history allometry in mammals was greatly expanded due to the trade-off between growth rate and life span. For plants, different evolutionary outcomes are possible depending on the shape of trade-off between the rates of niche construction and nutrient uptake ability [2]. Moreover, the trade-off can also decide the ecological consequences of biotic interactions, such as in the classic example on trade-offs between colonization and competition [62,64,74]. Spatial coexistence can arise from the appropriate three-way interspecific trade-offs among competition, colonization and niche construction [15]. In our model, the trade-off between the intensity of niche construction and colonization rate can lead to complex dynamics and spatial patterns. Future works should consider different shapes of trade-off, e.g. convex or concave shape.

The three-species cyclic game is a typical paradigm for exploring the role of population mobility in coexistence, where coexistence can be achieved from a low level of mobility [29]. Different intensities of niche construction in our model means different colonization rates and resource availability of each species, with both affecting the mobility of species. Therefore, our results also emphasize the role of mobility in maintaining diversity in systems of cyclic competition, with the disparity in mobility could threaten species coexistence [26]. Moreover, dispersal and biotic interactions can affect spatial synchrony which increases the extinction risk and undermines metapopulation persistence [75]. Density-dependent dispersal could benefit species persistence through desynchronized dynamics of local populations [76,77]. The self-organized spatial patterns generated here are dependent on mobility of interacting species and can undoubtedly affect spatial synchrony $[76,78]$. In food webs, the role of mobility on spatial synchrony could cause cascade effect through direct or indirect biotic interactions [79-81]. Here, spatial synchrony is also related to the symmetry breaking. When the three species have identical parameters, increasing niche-constructing intensity can not only transform the system dynamics from disorder to order, but also accelerate the event of extinction of the superior competitor. Once the symmetry is lost, the spatial pattern of rings can be easily disrupted. With increasing disparity between niche-constructing intensities, species distribution became static and fixed in space; that is, niche construction affects both the spatial synchrony and symmetry breaking in cyclic competition.

Extension of the classic cyclic competition from three species (or players) to more have been explored especially in cases modeling microbial communities [82] and large-scale ecological food webs and networks [83-88]. In general, the complexity of spatial pattern and dynamic behavior are drastically increasing with the number of species [27,29,33,34,36]. More investigations are needed for such player-rich cyclical systems, especially on the effects of network topology, mobility and interaction range [30]. Here, niche construction as an important regulating force for local adaptation, established a positive feedback between species and their environments [5,89]. Results from cellular automata have suggested that 'ecological imprint' can emerge through such a reinforcing feedback, forming fine-scale habitat heterogeneity [9,15], thus ensuring biodiversity maintenance via fostering sharp boundaries of species distributions $[9,10,15,90]$. In any case, $n$-player cyclic competition games with $n>3$, together with players affecting each other's niches, deserve further investigation.

Adaptive dynamics, a mathematical approach for studying evolutionary changes, is frequently used to discuss core problems in evolutionary biology, especial polymorphism and diversification through evolutionary branching (e.g. [91-94]). There is much less research about how adaptive divergence between closely related species can affect abiotic environmental conditions and about whether such effects can influence selection pressures so as to either promote or constrain further evolutionary divergence $[89,95]$. The methodology of adaptive dynamics could hold the key for future breakthrough in studying 
the effect of niche construction on evolution because of its concepts and techniques for modeling the dynamics of long-term phenotypic evolution.

\section{Acknowledgments}

This work was supported by the National Natural Science Foundation of China (nos. 31100308 and 11401115) to XH, the National Natural Science Foundation of China (no. 11501128) to BC and the National Research Foundation of South Africa (nos. 76912 and 89967) to $\mathrm{CH}$. We are grateful to Beverley Laniewski for her constructive comments on this manuscript. XH thanks China Scholarship Council and York Centre for Complex Systems Analysis of University of York for its hospitality. We are also grateful to the constructive comments from two anonymous reviewers and the editor.

\section{References}

[1] K.N. Laland, F.J. Odling-Smee, M.W. Feldman, The evolutionary consequences of niche construction: A theoretical investigation using two-locus theory, J. Evol. Biol. 9 (1996) 293-316

[2] G. Kylafis, M. Loreau, Ecological and evolutionary consequences of niche construction for its agent, Ecol. Lett. 11 (2008) 1072-1081

[3] G. Kylafis, M. Loreau, Niche construction in the light of niche theory, Ecol. Lett. 14 (2011) 82-90.

[4] F.J. Odling-Smee, K.N. Laland, M.W. Feldman, Niche Construction: The Neglected Process of Evolution, Princeton University Press, Princeton, 2003.

[5] F.J. Odling-Smee, D.H. Erwin, E.P. Palcovacs, M.W. Feldman, K.N. Laland, Niche construction theory: A practical guide for ecologists, Quaterly Rev. Biol. 88 (2013) 4-28.

[6] F.J. Odling-Smee, H.C. Plotkin, Niche constructing phenotypes, The Role of Behavior in Evolution, MIT Press, Cambridge, 1988, pp. 73-132.

[7] F.J. Odling-Smee, K.N. Laland, M.W. Feldman, Niche construction, Am. Nat. 147 (1996) 641-648

[8] X. Han, Z. Li, C. Hui, Y. Zhang, Polymorphism maintenance in a spatially structured population: A two-locus genetic model of niche construction, Ecol, Model. 192 (2006) 160-174.

[9] X. Han, C. Hui, Y. Zhang, Effects of time-lagged niche construction on metapopulation dynamics and environmental heterogeneity, Appl. Math. Comput. 215 (2009) 449-458.

[10] X. Han, C. Hui, Niche construction on environmental gradients: The formation of fitness valley and stratified genotypic distribution, PLoS ONE 9 (6) (2014) e99775.

[11] R.L. Day, K.N. Laland, F.J. Odling-Smee, Rethinking adaptation, Perspect. Biol. Med. 46 (2003) 80-95.

[12] K.N. Laland, F.J. Odling-Smee, M.W. Feldman, Niche construction: Do the changes that organisms make to their habitats transform evolution and influence nature selection? Nature 429 (2004) 609.

[13] J. Vandermeer, The importance of a constructivist view, Science 303 (2004) 472-474

[14] K.N. Laland, K. Sterelny, Seven reasons (not) to neglect niche construction, Evolution 60 (2006) 1751-1762.

[15] C. Hui, Z. Li, D. Yue, Metapopulation dynamics and distribution, and environmental heterogeneity induced by niche construction, Ecol. Model. 177 (2004) 107-118.

[16] X. Han, Y. Zhang, Dynamical analysis of niche construction in n-population metapopulation, Acta. Ecol. Sin. 28 (7) (2008) 3271-3276.

[17] K. Tainaka, Vortices and strings in a model ecosystem, Phys. Rev. E. 50 (1994) 3401-3409.

[18] M. Frean, E.R. Abraham, Rock-scissors-paper and the survival of the weakest, Proc. Biol. Sci. 268 (2001) 1323-1327.

[19] C.R. Johnson, I. Seinen, Selection for restraint in competitive ability in spatial competition systems, Proc. R. Soc. Lond. B 269 (2002) 655-663.

[20] P. Szabó, T. Czárán, G. Szabó, Competing associations in bacterial warfare with two toxins, J. Theor. Biol. 248 (2007) $736-744$

[21] G. Szabó, A. Szolnoki, R. Izsak, Rock-scissors-paper game on regular small-world networks, J. Phys. A Math. Gen. 37 (2004) 2599.

[22] T. Reichenbach, M. Mobila, E. Frey, Mobility promotes and jeopardize biodiversity in rock-paper-scissors games, Nature 448 (2007) $1046-1049$.

[23] T. Reichenbach, M. Mobila, E. Frey, Noise and correlation in a spatial population model with cyclic competition, Phys. Rev. Lett. 99 (2007) 238105.

[24] T. Reichenbach, M. Mobila, E. Frey, Self-organization of mobile population in cyclic competition, J. Theor. Biol. 254 (2008) $368-383$.

[25] W. Wang, X. Ni, Y. Lai, C. Grebogi, Pattern formation, synchronization, and outbreak of biodiversity in cyclically competing games, Phys. Rev. E. 83 (2011) 211-222.

[26] M.W. Adamson, A.Y. Morozov, Revising the role of species mobility in maintaining biodiversity in communities with cyclic competition, Bull. Math. Biol. 74 (2012) 2004-2031.

[27] S. Feng, C. Qiang, Self-organization of five species in a cyclic competition game, Physica A 392 (2013) 4675-4682

[28] B. Intoy, M. Pluming, Extinction in four species cyclic competition, J. Stat. Mech. 2013 (2013) P08011.

[29] H. Cheng, N. Yao, Z. Huang, J. Park, Y. Do, Y. Lai, Mesoscopic interactions and species coexistence in evolutionary game dynamics of cyclic competitions, Sci. Rep. UK 4 (2014) 7486, doi:10.1038/srep07486.

[30] A. Szolnoki, M. Mobilia, L. Jiang, B. Szczesny, A.M. Rucklidge, M. Perc, Cyclic dominance in evolutionary games: a review, J. R. Soc. Interface 11 (2014) 20140735

[31] B. Intoy, Pure and Mixed Strategies in Cyclic Competition: Extinction, Coexistence, and Patterns. (Doctoral Dissertation) (2015), Virginia Polytechnic Institute and State University. URL: http://hdl.handle.net/10919/51999.

[32] D. Grošelj, F. Jenko, E. Frey, How turbulence regulates biodiversity in systems with cyclic competition, Phys. Rev. E 91 (2015) 033009.

[33] M. Perc, A. Szolnoki, Noise-guided evolution within cyclical interactions, New J. Phys. 9 (2007) 267.

[34] M. Perc, A. Szolnoki, G. Szabó, Cyclical interactions with alliance-specific heterogeneous invasion rates, Phys. Rev. E 75 (2007) 052102.

[35] A. Szolnoki, Z. Wang, J. Wang, X. Zhu, Dynamically generated cyclic dominance in spatial prisoner's dilemma games, Phys. Rev. E 82 (2010) 036110.

[36] J. Vukov, A. Szolnoki, G. Szabó, Diverging fluctuations in a spatial five-species cyclic dominance game, Phys. Rev. E 88 (2013) 022123.

[37] A. Szolnoki, J. Vukov, M. Perc, From pairwise to group interactions in games of cyclic dominance, Phys. Rev. E 89 (2014) 062125.

[38] A. Szolnoki, M. Perc, Vortices determine the dynamics of biodiversity in cyclical interactions with protection spilliovers, New J. Phys. 17 (2015) 113033.

[39] D. Semmann, H.-J. Krambeck, M. Milinski, Volunteering leads to rock-paper-scissors dynamics in a public goods game, Nature 425 (2003) $390-393$.

[40] D.R. Amor, J. Fort, Effects of punishment in amobile population playing the prisoners dilemma game, Phys. Rev. E 84 (2011) 066115.

[41] A. Szolnoki, G. Szabó, M. Perc, Phase diagrams for the spatial public goods game with pool punishment, Phys. Rev. E 83 (2011) 036101.

[42] A. Szolnoki, M. Perc, G. Szabó, Defense mechanisms of empathetic players in the spatial ultimatum game, Phys. Rev. Lett. 109 (2012) 078701.

[43] C. Hauert, Replicator dynamics of reward \& reputation in public goods games, J. Theor. Biol. 267 (2010) $22-28$.

[44] A. Szolnoki, M. Perc, Reward and cooperation in the spatial public goods game, EPL 92 (2010) 38003.

[45] A. Szolnoki, M. Perc, Correlation of positive and negative reciprocity fails to confer an evolutionary advantage: Phase transitions to elementary strategies, Phys. Rev. X 3 (2013) 041021.

[46] P.W. Anderson, More is different, Science 177 (1972) 393-396.

[47] R. Li, B. Bowerman, Symmetry breaking in biology, Cold Spring Harb. Perspect. Biol. 2 (2010) a003475.

[48] R.D. Mullins, Cytoskeletal mechanisms for breaking cellular symmetry, Cold Spring Harb. Perspect. Biol. 2 (2009) a003392.

[49] J. Van der Gucht, C. Sykes, Physical model of cellular symmetry breaking, Cold Spring Harb. Perspect. Biol. 1 (2009) a001909.

[50] J. Dworkin, Cellular polarity in prokaryotic organisms, Cold Spring Harb. Perspect. Biol. 1 (2009) a003368. 
[51] F. Chang, S.G. Martin, Shaping fission yeast with microtubules, Cold Spring Harb. Perspect. Biol. 1 (2009) a001347.

[52] B.D. Slaughter, S.E. Smith, R. Li, Symmetry breaking in the life cycle of the budding yeast, Cold Spring Harb. Perspect. Biol. 1 (2009) a003384.

[53] S. Tahirovic, F. Bradke, Neuronal polarity, Cold Spring Harb. Perspect. Biol. 1 (2009) a001644.

[54] W.J. Nelson, Remodeling epithelial cell organization: Transitions between front-rear and apical-basal polarity, Cold Spring Harb. Perspect. Biol. 1 (2009) a000513.

[55] E.K. Vladar, D. Antic, J.D. Axelrod, Planar cell polarity signaling: The developing cell's compass, Cold Spring Harb. Perspect. Biol. 1 (2009) a002964.

[56] A.D. Barton, S. Dutkiewicz, G. Flierl, J. Bragg, M.J. Follows, Patterns of diversity in marine phytoplankton, Science 327 (2010) $1509-1511$.

[57] S.M. Burrows, T. Butler, P. Jöckel, H. Tost, A. Kerkweg, U. Pöschl, M.G. Lawrence, Bacteria in the global atmosphere-Part 2: Modeling of emissions and transport between different ecosystems, Atmos. Chem. Phys. 9 (2009) 9281-9297.

[58] F. Zhang, Z. Li, C. Hui, Spatiotemporal dynamics and distribution patterns of cyclic competition in metapopulation, Ecol. Model. 193 (2006) $721-735$.

[59] J.B.C. Jackson, L. Buss, Allelopathy and spatial competition among coral reef invertebrates, Proc. Natl. Acad. Sci. USA 72 (1975) $5160-5163$.

[60] B. Sinervo, C.M. Lively, The rock-scissors-paper game and the evolution of alternative male strategies, Nature 380 (1996) $240-243$.

[61] B. Kerr, M.A. Riley, M.W. Feldman, B.J.M. Bohannan, Local dispersal promotes biodiversity in a real-life game of rock-paper-scissors, Nature 418 (2002) $171-174$.

[62] D. Tilman, Competition and biodiversity in spatially structured habitats, Ecology 75 (1994) 2-16.

[63] K.N. Laland, F.J. Odling-Smee, M.W. Feldman, Evolutionary consequences of niche construction and their implications for ecology, P. Natl. Acad. Sci. USA 96 (1999) 10242-10247.

[64] R.H. MacArhur, E.R. Pianka, On optimal use of a patchy environment, Am. Nat. 100 (1966) 603-609.

[65] F. Zhang, C. Hui, Recent experience-driven behaviour optimizes foraging, Anim. Behav. 88 (2014) 13-19.

[66] D.W. Stephens, J.R. Krebs, Foraging Theory, Princeton University Press, Princeton, 1986.

[67] J.W. Silvertown, J.L. Doust, Introduction to Plant Population Biology, Blackwell, Oxford, 1993.

[68] C. Hui, Z. Li, Dynamical complexity and metapopulation persistence, Ecol. Model. 164 (2003) 201-209.

[69] C. Hui, Z. Li, Distribution patterns of metapopulation determined by Allee effects, Popul. Ecol. 46 (2004) 55-63.

[70] C. Hui, M.A. McGeoch, Does the self-similar species distribution model lead too unrealistic predictions? Ecology 89 (2008) $2946-2952$.

[71] C. Hui, M.A. McGeoch, Zeta diversity as a concept and metric that unifies incidence-based biodiversity patterns, Am. Nat. 184 (2014) 684-694.

[72] C. Hui, D. Yue, Niche construction and polymorphism maintenance in metapopulation, Ecol. Res. 20 (2005) 115-119.

[73] E.L. Charnov, The optimal balance between growth rate and survival in mammals, Evol. Ecol. Res. 6 (2004) 307-313.

[74] H.S. Horn, R.H. MacArthur, Competition among fugitive species in a harlequin environment, Ecology 53 (1972) 749-752.

[75] J. Ylikarjula, S. Alaja, J. Laakso, D. Tesar, Effects of patch number dispersal patterns on population dynamics and synchrony, J. Theor. Biol. 207 (2000) $377-387$.

[76] Z. Liu, F. Zhang, C. Hui, Density-dependent dispersal complicates spatial synchrony in tri-trophic food chains, Popul. Ecol. 58 (2016) 223-230.

[77] C. Hui, N. Roura-Pascual, L. Brotons, R.A. Robinson, K.L. Evans, Flexible dispersal strategies in native and non-native ranges: Environmental quality and the 'good-stay, bad-disperse' rule, Ecography 35 (2012) 1024-1032.

[78] O.N. Bjørnstad, J. Bascompte, Synchrony and second-order spatial correlation in host-parasitoid systems, J. Anim. Ecol. 70 (2001) $924-933$.

[79] F. Zhang, C. Hui, J.S. Terblanche, An interaction switch predicts the nested architecture of mutualistic networks, Ecol. Lett. 14 (2011) $797-803$.

[80] C. Hui, L.C. Foxcroft, D.M. Richardson, S. MacFadyen, L.C. Foxcroft, D.M. Richardson, P. Pysek, P. Genovesi, A cross-scale approach for abundance estimation of invasive alien plants in a large protected area, Plant Invasions in Protected Areas: Patterns, Problems and Challenges, Springer, Berlin, 2013, pp. 73-88.

[81] H.O. Minoarivelo, C. Hui, J.S. Terblanche, S.L. Kosakovsky Pond, K. Scheffler, Detecting phylogenetic signal in mutualistic interaction networks using a Markov process model, Oikos 123 (2014) 1250-1260.

[82] G. Szabó, T. Czárán, Phase transition in a spatial Lotka-Volterra model, Phys. Rev. E 63 (2001) 061904.

[83] S. Allesina, D. Alonso, M. Pascual, A general model for food web structure, Science 320 (2008) 658-661.

[84] S Allesina, J.M. Levine, A competitive network theory of species diversity, Proc. Natl. Acad. Sci. USA 108 (2011) 5638-5642.

[85] D.B. Stouffer, M. Sales-Pardo, M.I. Sirer, J. Bascompte, Evolutionary conservation of species' roles in food webs, Science 335 (2012) $1489-1492$.

[86] C. Hui, H.O. Minoarivelo, S. Nuwagaba, A. Ramanantoanina, P. Pontarotti, Adaptive diversification in coevolutionary systems, Evolutionary Biology: Biodiversification from Genotype to Phenotype, Springer, Berlin, 2015, pp. 167-186.

[87] S. Nuwagaba, F. Zhang, C. Hui, A hybrid behavioural rule of adaptation and drift explains the emergent architecture of antagonistic networks, Proc. R. Soc. B Biol. Sci. 282 (2015) 20150320.

[88] H.O. Minoarivelo, C. Hui, Trait-mediated interaction leads to structural emergence in mutualistic networks, Evol. Ecol. 30 (2016) 105-121.

[89] B.M. Matthews, L.D. Meester, C.G. Jones, B.W. Ibelings, T.J. Bouma, V. Nuutinen, J.V. Koppel, F.J. Odling-Smee, Under niche construction: An operational bridge between ecology, evolution, and ecosystem science, Ecol. Monogr. 84 (2014) 245-263.

[90] X. Han, Y. Huang, C. Hui, Spatial distributions of niche-constructing populations, Comput. Ecol. Softw. 5 (4) (2015) 286-298.

[91] J.A.J. Metz, R.M. Nisbet, S.A.H. Geritz, How should we define fitness for general ecological scenarios? Trends Ecol. Evol. 7 (1992) 198-202.

[92] M. Doebeli, U. Dieckmann, Evolutionary branching and sympatric speciation caused by different types of ecological interaction, Am. Nat. 156 (2000) S77-S101.

[93] F. Zhang, C. Hui, A. Pauw, Adaptive divergence in Darwin's race: How coevolution can generate trait diversity in a pollination system, Evolution 67 (2013) 548-560.

[94] P. Landi, C. Hui, U. Dieckmann, Fisheries-induced disruptive selection, J. Theor. Biol. 365 (2015) 204-216.

[95] J.B. Losos, Adaptive radiation, ecological opportunity, and evolutionary determinism, Am. Nat. 175 (2010) 623-639. 